\title{
A NOVEL FORM OF THERMO-ELECTRIC BATTERY.
}

BY O. J. REED.

If further proof were needed of the very high thermo-electric power of metals in contacts with electrolytes, a very striking and conclusive proof may be found in the following experiments.

The apparatus consists of a fused mass of caustic potash or soda maintained at a temperature of $500^{\circ}$ to $800^{\circ} \mathrm{C}$. into which two conducting rods are inserted, both of the same metal and as nearly alike as possible in every respect except form. One of the rods, marked в in Figs. 1 and 2, is of cylindrical form. The other, marked $\mathrm{A}$, is in the form of a cylinder having a deep recess cut or turned out near one end, leaving at the end a short cylindrical head attached to the rod by a very narrow stem.

In order to obtain two pieces of metal having, as nearly as possible, the same molecular structure and chemical composition, a uniform rod of Bessemer steel $\frac{5}{16}$ inch in diameter and two feet long, was cut in two equal parts. The severed ends which were originally together at the middle of the rod are turned smooth and flat to form the lower or contact ends of the rods, A and B, shown in Figs. 1 and 2. The head formed on the end of A is $\frac{1}{4}$ inch long and the stem by which it is attached is half an inch long and $\frac{1}{20}$ inch in diameter. The two rods thus prepared are held parallel to each other and about $\frac{1}{4}$ inch apart in a wooden clamp with their ends in the same plane at right angles to their axes.

The rods are now inserted in a vertical position to a depth of $\frac{1}{2}$ inch into the fused caustic alkali. The surface of the fused alkali should come above the head of the rod, $\mathrm{A}$, but not above the stem, as shown in Fig. 2. The cup used in this experiment to 
hold the molten alkali is of sheet steel about two inches in diameter and three inches deep. The rods are inserted in the center of the cup, in order that the temperature and chemical composition of the mass of alkali immediately surrounding the rods, as well as the temperature of the air surrounding them may be as nearly homogeneous as possible. By these precautions all effects of every nature are eliminated, except such as may be due to the difference in temperature between the submerged ends of the two rods caused by their difference in form, which enables the rod, $\mathrm{s}$, to get rid of its communicated heat by conduction more rapidly than the rod, $A$. The temperature of the alkali is maintained at about $700^{\circ} \mathrm{C}$. After the rods have remained in the melted alkali a fow minutes and the room has beon darkened,

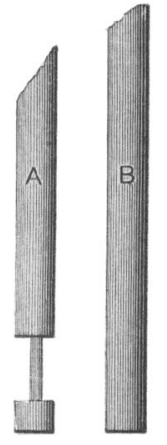

FIG. 1.

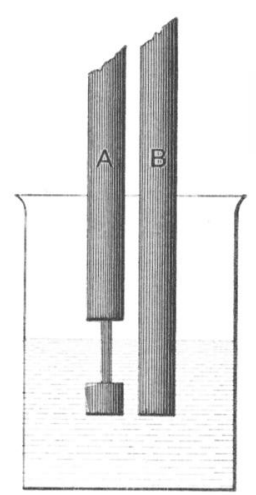

Fig. 2.

they are quickly withdrawn for inspection. The cylindrical head on the rod, $\mathrm{A}$, is seen to be at a uniform bright red heat, while the rod, B, is only perceptibly dull red at the extreme end. The difference in temperature between the cylindrical head of $\mathrm{A}$ and the hottest part of $\mathrm{B}$ is probably not less than $100^{\circ} \mathrm{C}$. The reason for this difference in temperature is apparent. The narrow stem of $\mathrm{A}$ conducts heat away very slowly, while the rod, B, of uniform diameter conducts away its heat with a rapidity many times greater. Assuming that both rods receive heat from the surrounding liquid initially at the same rate per unit of surface, the head of rod, $A$, must become much hotter than any part of $\mathrm{B}$, since it has a relatively larger surface of contact with the communicating liquid and loses its heat by conduction much more slowly. 
In this arrangement we have two pieces of metal as nearly alike as possible in chemical composition and molecular structure, subjected to the same conditions in all respects, except that the temperature of one at its junction with the electrolyte is higher than the temperature of the other at its junction with the electrolyte. In other words, the electrolyte has one hot and one cold junction with pieces of the same metal inserted in it to the same depth and subjected as far as known to no other disturbing influences.

This arrangement gives an electromotive force, varying with the temperature and constitution of the electrolyte between -0.2 volt and +1 volt. The difference in temperature between the two junctions evidently increases with the temperature of the fused alkali, being zero when the temperature of the communieating liquid is the same as that of the surrounding air. The difference in temperature between the junctions increases rapidly for a certain period from the beginning of the experiment, while the rod, $\mathrm{B}$, is comparatively cold and capable of dissipating heat rapidly and while the temperature of $\mathrm{A}$ is rapidly rising. Following this will be a period, during which the difference in temperature between the junctions will diminish until both rods attain throughout their entire lengths the maximum temperatures they are capable of attaining under the conditions.

I have previously shown ${ }^{1}$ some of the peculiar variations of electromotive force under changes of temperature and alterations in the constitution of the electrolyte, which are exhibited in a cell consisting of an iron cup containing fused alkali as one electrode and an iron or other conducting rod inserted in the alkali as the other electrode. In that apparatus the iron cup imparted heat to the alkali and the alkali imparted heat to the rod. From this circumstance it follows that the junction of the electrolyte with the iron cup is necessarily hotter than the junction of the electrolyte with the rod. The cup, being the hotter of the two pieces of iron in that experiment, should act in the same qualitative manner as the rod, $A$, in the experiment illustrated in Fig. 2 above, a being the hotter of the two rods. But the difference in temperature between the two rods, $\mathrm{A}$ and $\mathrm{B}$, can never be as great as the difference between that of the heated cup and that of the rod, в. These conclusions are entirely con- 
firmed by the experiment, the rod, A, acting in the same manner as the cup, with the exception that the electromotive force obtained from it is not so great.

In attempting to explain the electromotive force developed between the heated cup and the inserted rod as a result of chemical action, some have resorted to the argument that the upper layers of the liquid may have an affinity for iron different from that of the lower layers, assuming that oxidation of iron in the upper layers causes reduction of iron in the lower layers and at the same time evolves electrical energy. The experiment with the two rods inserted to equal depths evidently leaves no room for such an argument, aside from its inherent absurdity. As a matter of fact there is no evidence of the existence of any such layers of varying chemical composition, the entire mass being continually stirred up by rapid convection currents like boiling water. The same results are obtained even when the rods extend to within a millimeter of the bottom of the cup. I have also obtained an electromotive force of nearly one volt between the cup and an inserted iron rod when the rod actually rested on the bottom of the cup, metallic contact being prevented only by a thin film of oxide or of the electrolyte.

Admitting that the chemical affinity between hot iron and the electrolyte is undoubtedly different from that between cold iron and the electrolyte, no energy could be evolved in any form due to this differential affinity, except energy derived from the heat of the furnace, since the existence of this differential affinity depends only upon the difference in temperature. To suppose that the electrical energy of this cell is derived from chemical action, involves the assumption that iron has a greater affinity than iron for oxygen, or that oxygen will select and combine with one of two similar pieces of iron while it dissociates from the other, and the further assumption that such a process will evolve energy. If this is possible, it can be possible only because of the difference in temperature, which in turn can be maintained only by a constant expenditure of heat from the furnace. Otherwise we should have an inexhaustible source of energy. If oxygen can evolve energy by leaving one piece of iron and combining with another, it may, with equal reason, leave the second and re-combine with the first, evolving an equal amount of energy, and there would be no limit to the number of such reversals, all ovolving energy. 
The experiment shows that the rods, $\mathrm{A}$ and $\mathrm{B}$, evolve electrical energy and exhibit a high electromotive force under circumstances that are identical in all respects except temperature, and that the action is, therefore, thermo-electric.

The experiment may be varied by using two rods in the form of $\mathrm{B}$. In this experiment there is no evidence of electromotive force when the rods are in a vertical position, so that both are equally heated by the electrolyte, as shown in Fig. 3. Upon inclining the rods so that one barely makes contact with the electrolyte, while the other extends about a quarter of an inch below the surface, as shown in Fig. 4, the rods acquire a considerable difference in temperature, which is clearly indicated to the eye, one being distinctly red and the other black. At the same time an electromotive force is developed, which in some

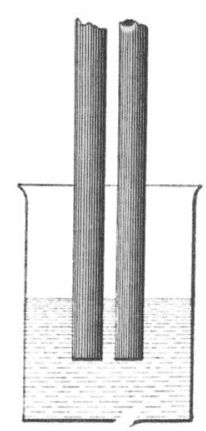

FIG. 3.

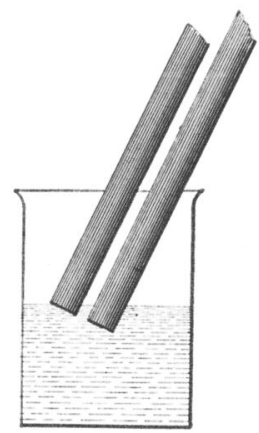

FIG. 4.

experiments has reached as high as 0.6 volt. Upon reversing the position of the rods, so that the hot one becomes cold and the cold one hot, the direction of the electromotive force slowly reverses. This reversal I have found may be repeated indefinitely, provided the other conditions of the experiment are maintained.

Rods of carbon, copper and various other metals may be substituted for the iron rods, and other electrolytes substituted for the canstic alkali. In all cases similar results, differing only in degree, are obtained. Many of these results are highly interesting and will be described later in detail.

The changes in the constitution of the alkali hydrate, which take place during its gradual dehydration have not yet been definitely determined. It is quite certain that these changes have a marked effect on the thermo-electric power of the cell. 
If any current be allowed to flow, the electrolytic action of the current immediately causes the formation of oxide of iron at one electrode and the reduction of previously existing oxide of iron at the other electrode. This necessarily produces a change of chemical composition in the electrolyte adjacent to the electrodes or on the surface of the electrodes themselves.

As long as oxide of iron remains dissolved in the electrolyte and in contact with the electrodes in sufficient quantity to transmit the current, the electrolytic action will neither absorb nor evolve energy, since the amount of iron oxidized at one electrode will be equal to the amount reduced at the other. The resistance of the electrolyte as a conductor only will cause a fall of the potential difference on closed circuit. If no oxide of iron is present in a dissolved condition in the electrolyte and if there is none on the surface of the hydrogen electrode available for reduction, the current can no longer flow without reducing some other constituent of the electrolyte, such as sodium or hydrogen. In other words, a counter-electromotive force or "polarization" ensues.

It was found by Deville ${ }^{1}$ that sodium hydrate does not decompose at any temperature into water and sodium oxide, but at about $1100^{\circ} \mathrm{C}$. it decomposes into sodium, hydrogen and oxygen. If, therefore, a condition should ensue, in which no oxide of iron is available for reduction while the current continues to flow and ferric oxide continues to form at the oxygen electrode, the electro-chemical reaction would be

$$
3 \mathrm{NaOH}+2 \mathrm{Fe}=\mathrm{Fe}_{2} \mathrm{O}_{3}+3 \mathrm{Na}+3 \mathrm{H} \text {. }
$$

This reaction could take place electrolytically at ordinary temperatures by the absorption of 115,000 calories, corresponding to a counter-èlectromotive force of $\frac{115,000}{6 \times 23,240}=0.82$ volt. At the temperature of $500^{\circ} \mathrm{C}$., at which the thermo-electromotive force of the cell is about 1 volt, the reaction would absorb approximately 72,000 calories, corresponding to a counterelectromotive force of $\frac{72,000}{6 \times 23,240}=0.52$ volt.

There is reason to believe that this condition of the electrolyte does actually ensue at a certain stage of the experiment when the circuit is closed, and under certain conditions metallic sodium may be evolved in considerable quantities at the inner surface of 
the cup. The maximum quantity. of iron that can be dissolved in the fused caustic alkali is very small, and this appears to be held in solution only while there is present more water than corresponds to the formula for the alkali hydrate. This excess of water is driven off very slowly, the last trace leaving only at a temperature above $500^{\circ} \mathrm{C}$.

Liebenow and Strasser ${ }^{1}$ found that when the alkali, fused in an iron cup, reaches a temperature of about $550^{\circ} \mathrm{C}$. after prolonged heating, it undergoes a radical change in properties, changing color from green to dark brown and simultaneously absorbing heat and causing its own temperature to be temporarily reduced. With this change in the electrolyte the electromotive force undergoes a sudden change of about one volt.

The absorption of heat and consequent reduction of temperature can be accounted for only by a chemical change in the electrolyte which causes the heat to become latent. Such a reaction may be explained on the supposition that the electrolyte changes at this temperature from an aqueous solution to a dehydrated fused electrolyte. While water is present, the iron probably remains in solution as ferric acid (the highest known oxide of iron) in combination with the alkali as potassium or sodium ferrate, which in the highly concentrated solution has a green color if any sulphur is present. When the water which holds this salt in solution is finally driven out, the ferric acid, being insoluble in the dehydrated alkali hydrate, is decomposed into ferric oxide, potassic oxide and oxygen, the reaction being $2 \mathrm{~K}_{2} \mathrm{FeO}_{4}=2 \mathrm{~K}_{2} \mathrm{O}+\mathrm{Fe}_{2} \mathrm{O}_{3}+3 \mathrm{O}$.

The insoluble ferric oxide precipitated in the solid state by this reaction gives the dark brown color to the mass, which was observed by Liebenow and Strasser. This precipitate is found disseminated through the solidified hydrate on cooling. The dissociation of the ferric acid should be accompanied by the absorption of considerable heat-probably at that temperature about 150,000 calories. This would be sufficient to account for the remarkable fall of temperature, observed by Liebenow and Strasser to accompany the change of color from green to brown. A further examination of the results obtained by these investigators reveals the fact that this fall of temperature occurred only in those experiments in which either iron or nickel was present in the electrolyte.

1 Zeitschrift fur Elektrochemie, Feb. 20, 1897. 
The experiments referred to of Liebenow and Strasser are a series of elaborate in restigations on a number of alkaline thermoelectric cells, the results of which are entirely in accord with the explanation given above, and form the basis on which that explanation is chiefly founded. I am unable to agree with those investigators, as before stated, in some of their conclusions; as, for instance, the conclusion that electrical energy or any other form of energy can be evolved from the "passivity" of a metal

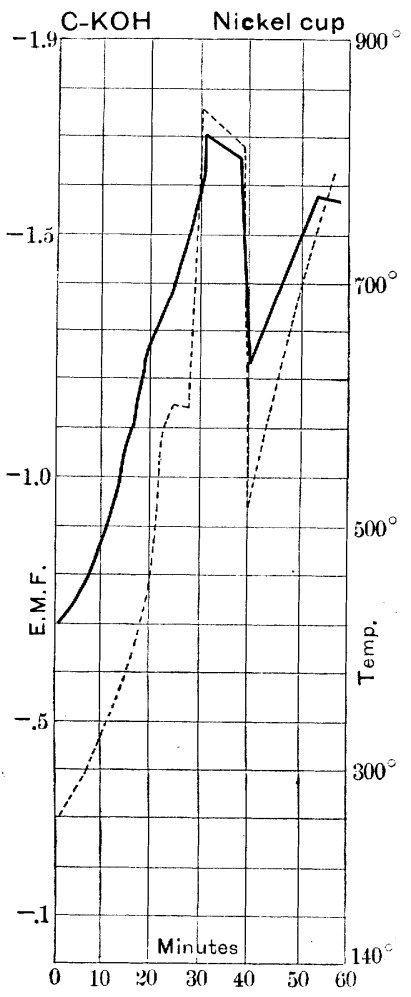

FIG. 5.

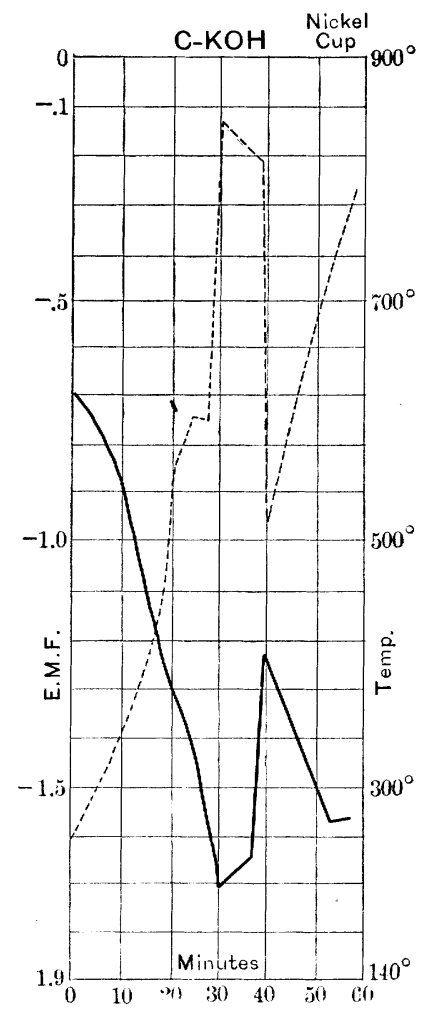

FIG. 6.

or from any other property of matter, and I believe the experiments they have described do more towards disproving their own preoonceived theory than any experimental work that has been done relating to this subject.

In those experiments the effects produced by various alterations of the electrolyte on the potential difference between various electrodes and an arbitrary " normal electrode" were determined. Simultaneous measurements of electromotive force and tem- 
perature were made which throw much light upon the nature of these cells. Unfortunately no measurements were made to determine at the same time the difference of temperature between the various electrodes.

The relation between the temperature of the electrolyte and the electromotive force of the carbon-caustic-potash cell, as indicated by the results of Liebenow and Strasser, is well illustrated in the curves shown in Fig. 5. In this diagram the abscissa represents the time measured from the beginning of the experiment, and the corresponding ordinate of the dotted line represents the temperature, while the ordinate of the full line represents the simultaneous difference of potential between the carbon rod and the "normal electrode." Liebenow and Strasser apparently failed to see the relation between the curves of temperature and electromotive force, simply because the direction of this electromotive force happened to be opposite to that which they had been arbitrarily measuring as increasing towards the top of the diagram. They, therefore, measured electromotive force downwards and the corresponding temperatures upwards and obtained the diagram shown in Fig. 6, which is not quite so easily interpreted. They apparently failed to get any meaning from it, merely remarking that it did not agree with the formula they had empirically deduced.

When the intensity of the electromotive force is compared directly with the temperature, instead of inversely, the relation becomes apparent, as shown in Fig 5. In this particular case the diagram shows decidedly that the electromotive force increases and decreases with the temperature of the electrolyte. From the nature and arrangement of the apparatus we know that the difference of temperature between the electrolyte and the rod will also increase and decrease with the temperature of the electrolyte. This dependence of electromotive force upon difference of temperature is shown in nearly all the diagrams of Liebenow and Strasser, when the electromotive force, whether positive or negative, is measured in the same direction as the temperature. And it must be so measured in order to make a direct comparison. The polarity or direction of the electromotive force has no particular bearing on the relation between its intensity and variations in temperature, but is dependent merely on the accidental position of the neutral point on the thermo-electric diagram. 
When any thermo-electric couple is heated up from a very low to a very high temperature without any great change in the difference of temperature between the hot and cold junctions, the electromotive force will diminish with increase of temperature until the neutral point is reached, where it will become zero and change sign. From that point it will increase with the temperature if the difference in temperature between the junctions does not diminish.

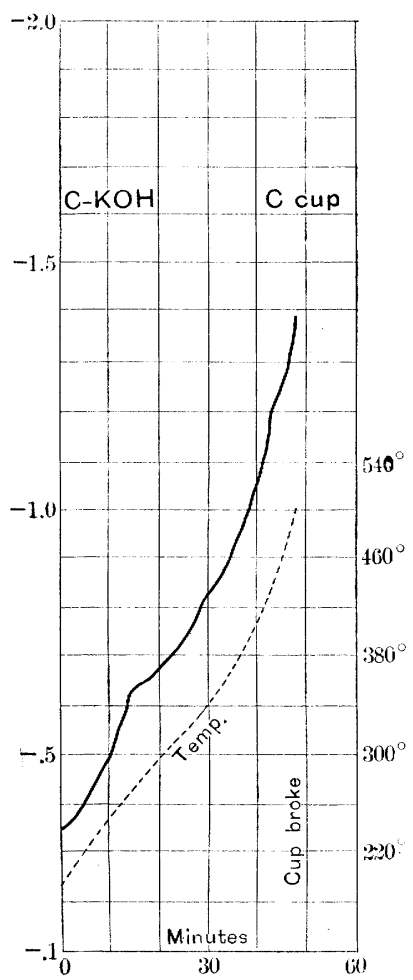

Fig. 7.

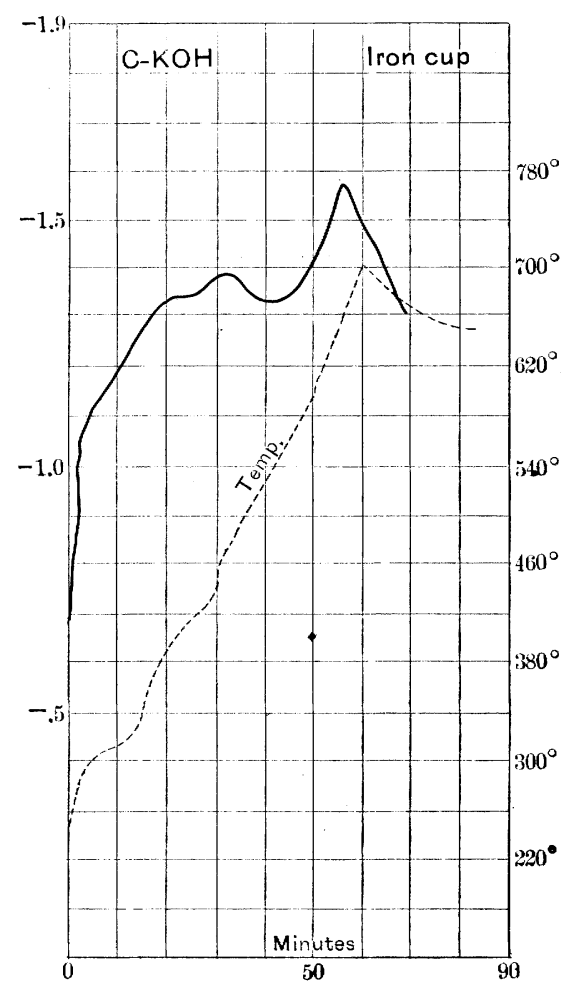

FIG. 8.

In the case shown in Fig. 5 it is evident that the electromotive force does not change sign. This means only that in this particular case (carbon-caustic-potash junction) the neutral point, if there be any, lies outside of the range of temperatures employed in this experiment, that is, the neutral point does not lie between $260^{\circ}$ and $850^{\circ} \mathrm{C}$.

When these facts are all taken into account the results obtained by Liebenow and Strasser, as far as they go, show that the 
electromotive force was produced by and depended upon the difference of temperature between the rod and the electrolyte, except in those cases where they purposely introduced disturbing elements. In such cases they do not describe the details of their apparatus and modus operandi sufficiently to enable us to judge what effect upon the temperature of various parts of the apparatus may have been produced by the various gases they introduced in some of their experiments and by closing the crucible with a

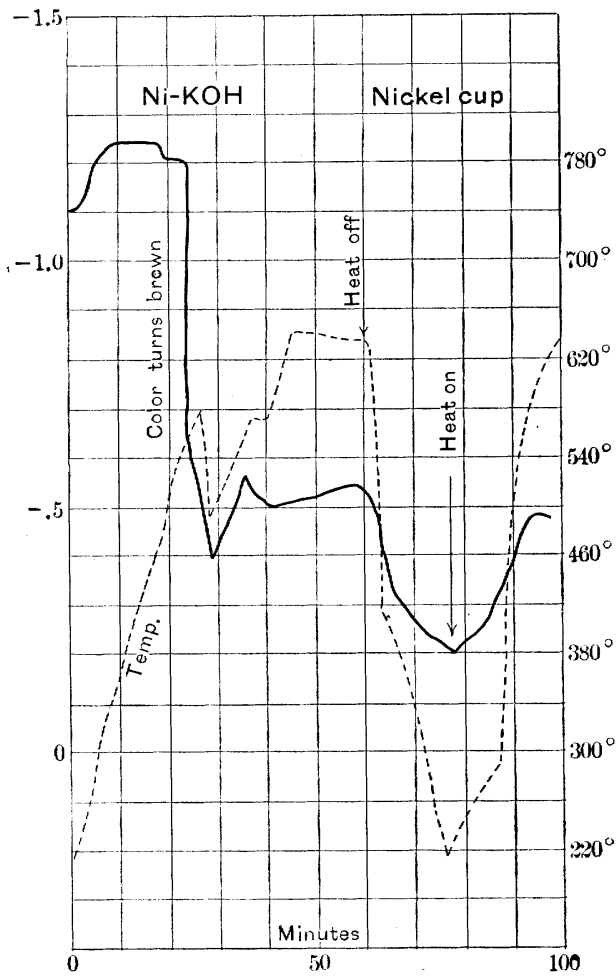

Fig. 9.

cover, which would naturally tend to keep the temperature of the rod and that of the electrolyte more nearly alike at the point of contact.

The experiment with the carbon rod and caustic potash was varied by Liebenow and Strasser by substituting for the nickel cup used in Fig. 5, in one case a carbon and in another case an iron cup. The results are exhibited (by inverting the curves of Liebenow and Strasser) in Figs. 7 and 8 respectively, which agree 
with Fig. 5 in showing that the curve of electromotive force in the carbon-caustic-potash couple follows in general the curve of temperature, and that the neutral point of this couple does not lie between $200^{\circ}$ and $700^{\circ} \mathrm{C}$.

A second group of most instructive experiments with metal rods substituted for the carbon rod (the results of which are exhibited in Figs. 9, 10, 11 and 12) demonstrate in an equally convincing manner that a change in the temperature of the electrolyte produces a corresponding change in the electromotive

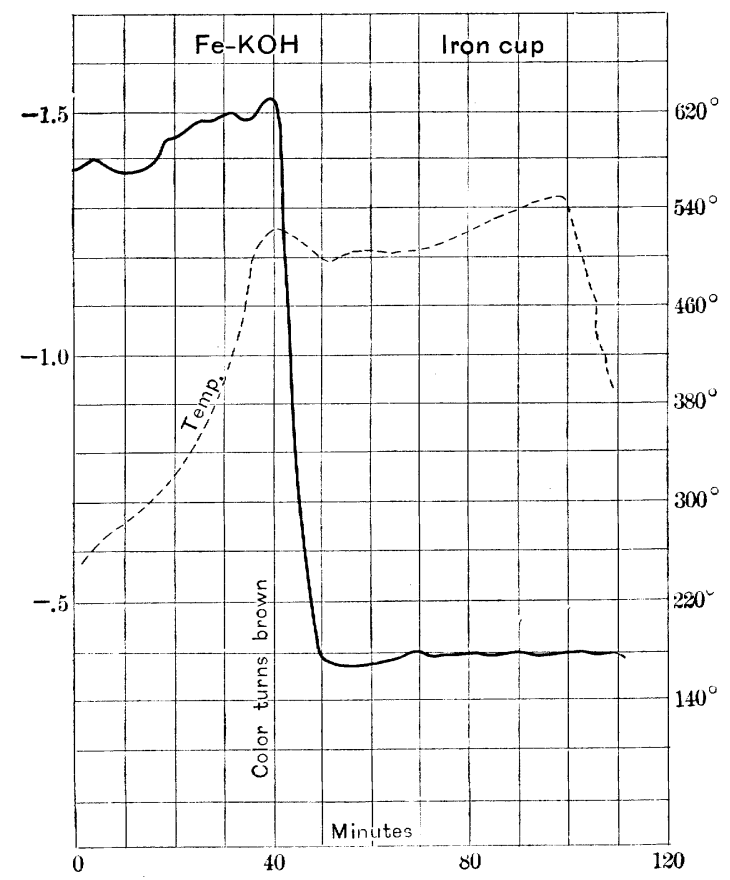

FIG. 10.

force. These curves were also inverted on the temperature curves in the diagrams of Liebenow and Strasser. In Fig. 9, showing the result when a nickel rod and cup are used, there are no less than eight well marked points of flexure in the temperature curve, each corresponding to a similar point in the curve of electromotive force. Fig. 11 differs from Fig. 10 only in the substitution of caustic soda for caustic potash.

An examination of these four curves shows some very instructive coincidences, among which are the following: 
1. The electromotive force increases with increasing temperature from the beginning of the experiment up to the moment the brown color of the electrolyte appears, indicating a chemical change and probably a condition of dehydration. This change was not marked in Fig. 11 by Liebenow and Strasser, but the curves of electromotive force and temperature both show that it must have occurred at the end of 26 minutes.

2. When this point is reached there is a sudden and very great change in electromotive force.

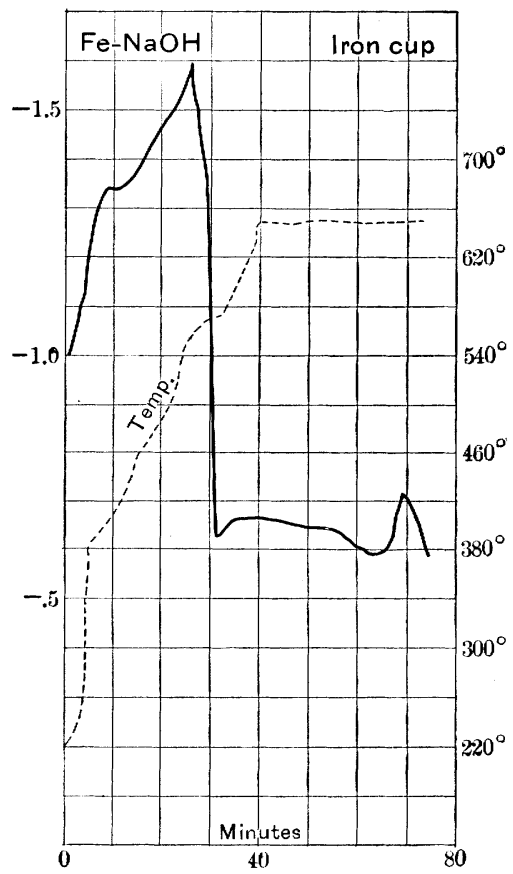

Fig. 11.

3. Simultaneous with this change of electromotive force and change of color, an absorption of heat occurs in the electrolyte, which causes a sudden reduction of its temperature. In Fig. 11 this reduction of temperature is indicated only by a flattened portion of the temperature curve. This reduction in the temperature of the electrolyte would necessarily greatly reduce the difference between its temperature and that of the metallic rod to which it communicates heat. It is conceivable that in some cases, where the absorption is very marked and sudden, heat 
may actually flow back from the rod to the electrolyte. This sudden reduction in the temperature of the electrolyte accounts satisfactorily for the change of electromotive force accompanying it.

4. From this point to the end of the experiment the curve of electromotive force follows in general the temperature curve.

In all cases except that of silver, Fig. 12, the temperature at which the sudden change occurs, lies between $524^{\circ}$ and $580^{\circ} \mathrm{C}$. The more rapidly the heat is applied, the higher is the temper-

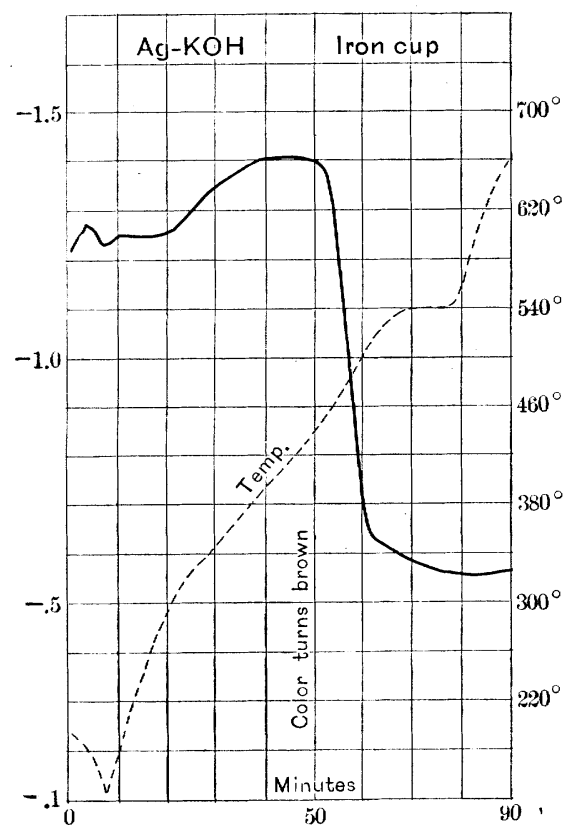

Fig. 12.

ature at which this change occurs and the more nearly vertical is the curve of electromotive force at that point. In Fig. 9 the dehydration point was reached in abont 23 minutes and the temperature attained before the change was $580^{\circ}$. In Fig. 11 the time was about 26 minutes and the temperature attained $570^{\circ}$. In Fig. 10 the time was 40 minutes and the temperature $524 .^{\circ}$

In Fig. 12 the time was 50 minutes, the temperature $460^{\circ}$, and no reduction of temperature was observed to accompany the change of color. This may be accounted for on the supposition 
that, owing to the slowness of the reaction in this case the continuous application of external heat was sufficient to prevent an actual fall of temperature.

In Fig. 13 the conditions were the same as those of Fig. 12, excepting that a silver cup was substituted for the iron cup and, consequently, there was no iron present in the electrolyte. The marked irregularities in the curve of electromotive force shown in Fig. 13 are to be expected from the high thermal conductivity of the silver cup and rod, which makes the apparatus very sensitive to fluctuations in the source of heat. The absence of

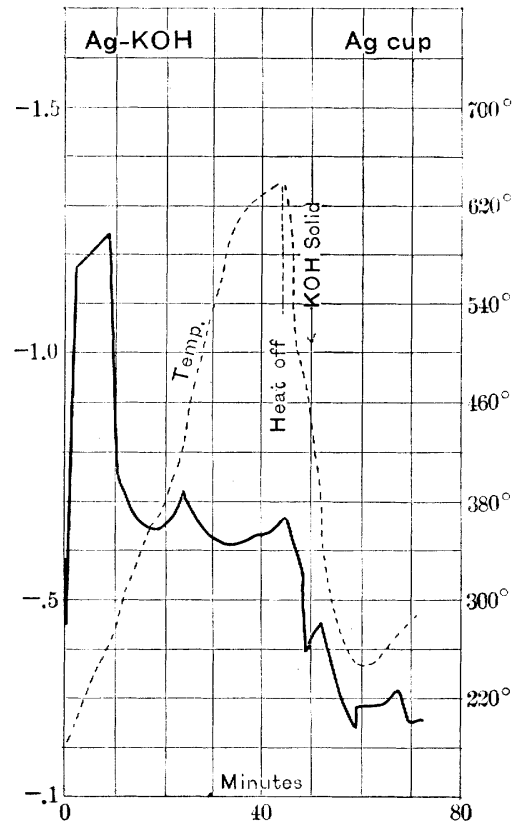

FIG. 13.

any indication of chemical change or change of color in the electrolyte or of any corresponding absorption of heat or change of electromotive force is accounted for by the fact that there is no iron or nickel in the solution and that the dehydration is accomplished without any chemical change due to the presence of impurities.

Figs. 14 and 15 show the result of substituting for the normal electrode in Figs. 8 and 6 respectively, rods of iron and nickel. The effect of this change was to remove the arbitrary zero of potential established by the introduction of the normal electrode 
and to transfer the neutral point, at which the electromotive force changes sign, to a position within the diagram. The neutra! point on these diagrams corresponds to the temperature of $555^{\circ} \pm 5^{\circ}$, coinciding with the temperature at which the chemical change and the absorption of heat occur. These curves are not inverted, but are copied without change from the curves given by Liebenow and Strasser.

In Figs. 16 and 17 the results are further complicated by the

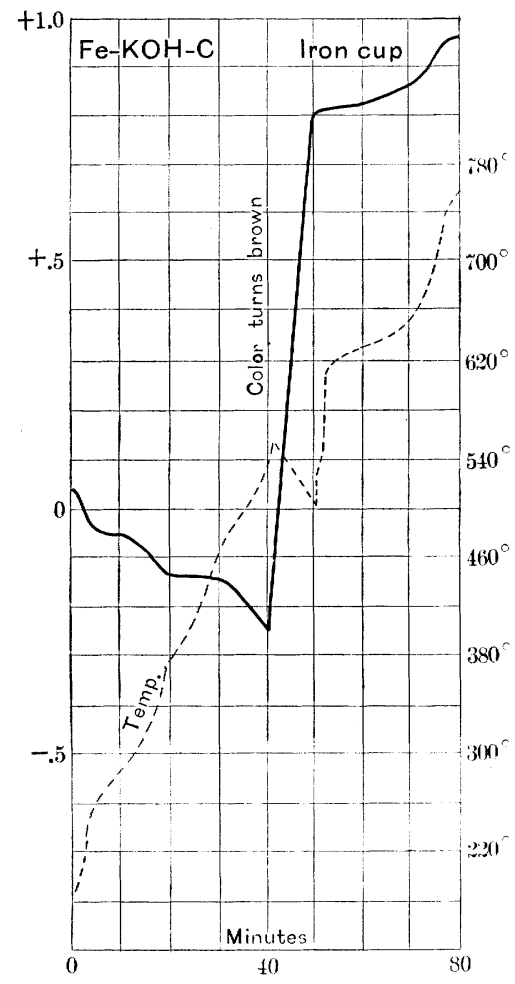

FIG. 14.

ase of various disturbing influences. The most important change introduced in these experiments was to close the cup with a practically air-tight cover, through which a carbon and an iron rod were inserted. This entirely prevented the circulation of air over the surface of the electrolyte and around the lower ends of the rods. The temperature of such a closed chamber above the electrolyte would be only slightly less than that of the electrolyte itself after the apparatus had become thoroughly 
heated. The lower ends of the carbon and iron rods, being surrounded by an atmosphere nearly as hot as the electrolyte, would have little difference in temperature at their surfaces of contact with the electrolyte, nothwithstanding their great difference in conductivity. Such an arrangement would result in a very much lower electromotive force. Liebenow and Strasser reported no experiments under exactly these conditions, but gave in Figs. 16 and 17 the results obtained by various moditications of these

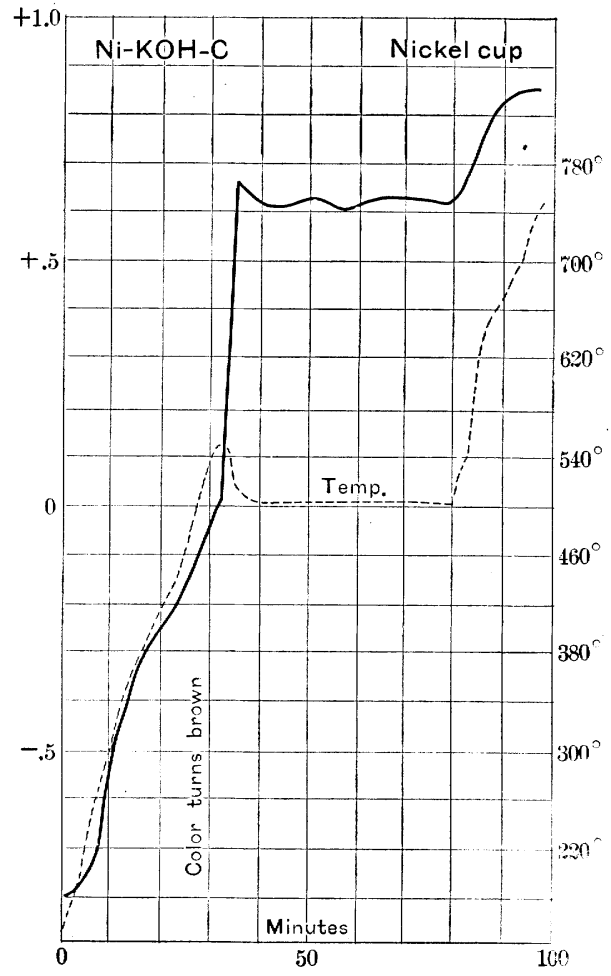

FIG. 15.

conditions, viz., by passing through the closed chamber various gases.

Unfortunately sufficient attention was not given to the details of these experiments to enable us to decide to what extent, if any, the results were due to the chemical action of the gases introduced, and to what extent they were due to their cooling effect. If the air and all the various gases were passed through the closed chamber at the same temperature and all with the 


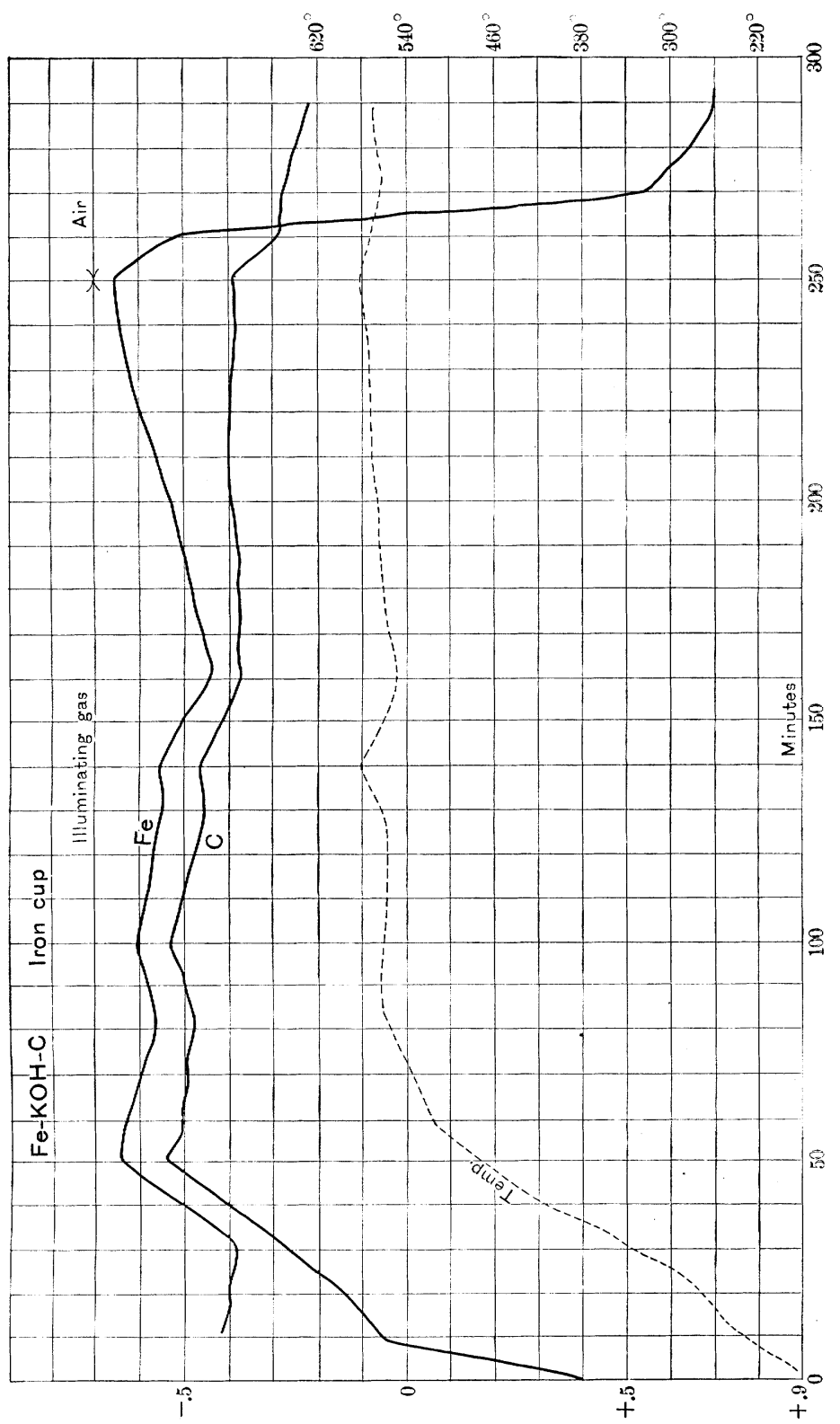

FIG. 16.

same velocity, the only difference in their cooling effects would be that due to the differences in their specific heats. These 
could not be very great and would probably be negligible. If, on the other hand, the gases were passed through the cell with greatly varying velocities, their cooling effects, particularly on the iron rod, would be very different.

Fig. 16 shows the result obtained by passing illuminating gas through the cell for four hours and ten minutes, then replacing it by air. At the end of the first hour the temperature and the electromotive force became practically constant and remained so until the change was made from illuminating gas to air. When

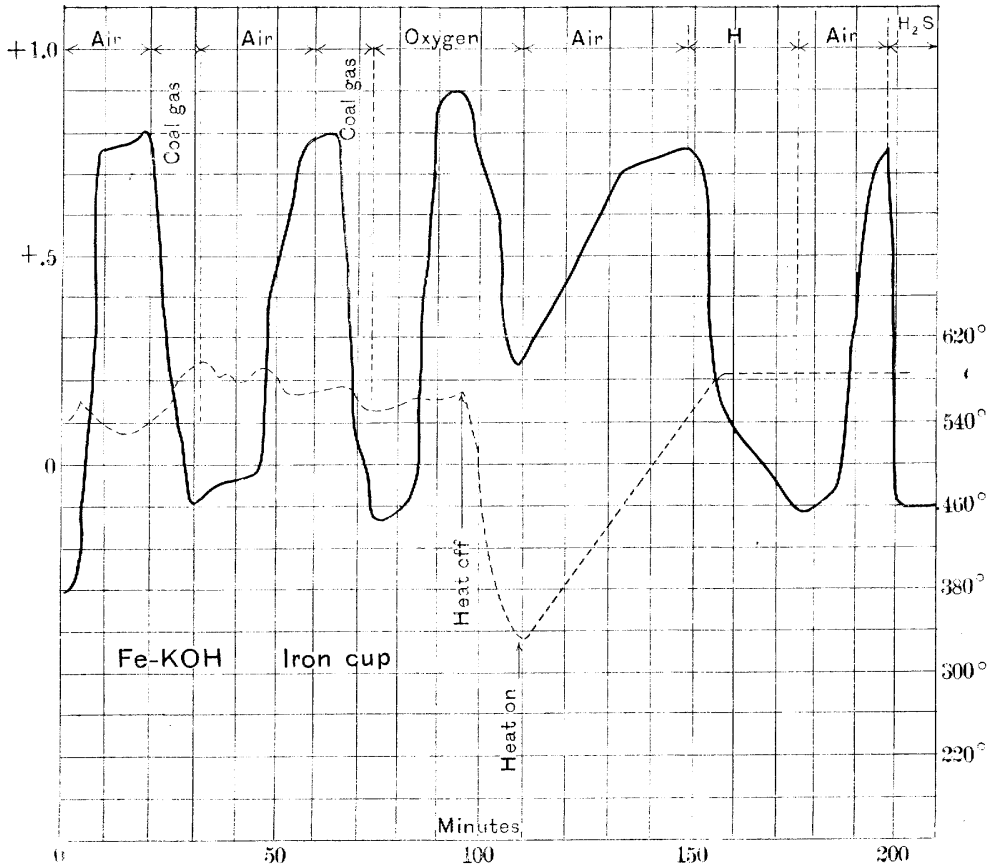

FIt. 17.

this change was made there was a noticeable, though not great fall in the temperature of the electrolyte, a corresponding change in the electromotive force of both the carbon and the iron as compared with the normal electrode, the change in the iron being very great. These curves, which are also the inverted curves of Liebenow and Strasser, are seen to follow the temperature curve in exactly the same manner as the curves of carbon and iron shown in Figs. 5 to 12.

In the absence of any statement to the contrary by Liebenow 
and Strasser the inference is reasonable that the air forced through the cell was in a large or unlimited quantity, while the flow of the combustible gases was adjusted to make the issuing jet burn with as small a flame as possible. If such were the case, the cooling effect of the air on the rods could not fail to produce the result shown in Fig. 16. The same explanation applies with equal force to the results shown in Fig. 17, in which various gases were successively passed through the cell. There is no doubt that the results are also complicated by various chemical changes resulting from the gases introduced. The introduction of pure oxygen, for example, in contact with the redhot carbon would certainly result in a vigorous combustion of the carbon, which would tend to increase its temperature even above that of the electrolyte and augment the effect shown in Fig. 17. But the lack of more detailed information concerning these experiments makes it useless to speculate further.

\section{Discussion.}

Mr. Enward P. Thompson:-I have been considering whether the proposition Mr. Reed has put before us has been strengthened by him by a variety of experiments. As I understand from his remarks, the electromotive force is due to the difference of temperature between the two electrodes. I was thinking that if that is what he considers it to be due to, it ought to be proved not merely by one experiment, but by several,- - that is, several kinds of experiments. He arranges two electrodes so that one becomes hotter than the other by the heated electrolyte, and of course, in that case, there is not such a very great difference of temperature. I should think that he might obtain a great difference by some outside means. For example, suppose he should heat the electrode $\mathrm{A}$ by an outside furnace, so as to get it to a great deal higher temperature, and then insert it, and see whether the electromotive force has increased; or reverse the experiment by heating the electrode $B$, so that it is about as hot as electrode $\mathrm{A}$, and see whether the electromotive force is zero. I am inclined to agree with Mr. Reed's presentation, but at the same time more than one kind of experiment should be in evidence.

It might be argued, although not on very solid grounds, that the reason why there is an electromotive force, is because there is less surface on one electrode than there is on the other. Now in Fig. 3, the two surfaces are equal, and there is no electromotive force. In Fig. 4 again the two surfaces are unequal and there is an electromotive force; so that a person, merely as a matter of argument, could say that the relative amounts of surface of the 